Honam Mathematical J. 34 (2012), No. 3, pp. 311-326

http://dx.doi.org/10.5831/HMJ.2012.34.3.311

\title{
A FURTHER GENERALIZATION OF APOSTOL-BERNOULLI POLYNOMIALS AND RELATED POLYNOMIALS
}

\author{
R. Tremblay, S. Gaboury* and J. Fugère
}

\begin{abstract}
The purpose of this paper is to introduce and investigate two new classes of generalized Bernoulli and Apostol-Bernoulli polynomials based on the definition given recently by the authors [29]. In particular, we obtain a new addition formula for the new class of the generalized Bernoulli polynomials. We also give an extension and some analogues of the Srivastava-Pintér addition theorem [28] for both classes. Finally, by making use of the new addition formula, we exhibit several interesting relationships between generalized Bernoulli polynomials and other polynomials or special functions.
\end{abstract}

\section{Introduction}

The generalized Bernoulli polynomials $B_{n}^{(\alpha)}(x)$ of order $\alpha \in \mathbb{C}$ and the generalized Euler polynomials $E_{n}^{(\alpha)}(x)$ of order $\alpha \in \mathbb{C}$, each of degree $n$ as well as in $\alpha$, are defined respectively by the following generating functions (see,[6, Vol.3, p. 253 et seq.] and [10, Section 2.8]):

$$
\left(\frac{t}{\mathrm{e}^{t}-1}\right)^{\alpha} \cdot \mathrm{e}^{x t}=\sum_{k=0}^{\infty} B_{k}^{(\alpha)}(x) \frac{t^{k}}{k !} \quad\left(|t|<2 \pi ; 1^{\alpha}:=1\right)
$$

and

$$
\left(\frac{2}{\mathrm{e}^{t}+1}\right)^{\alpha} \cdot \mathrm{e}^{x t}=\sum_{k=0}^{\infty} E_{k}^{(\alpha)}(x) \frac{t^{k}}{k !} \quad\left(|t|<\pi ; 1^{\alpha}:=1\right),
$$

where $\mathbb{C}$ denotes the set of complex numbers.

Received March 29, 2012. Accepted June 11, 2012.

2000 Mathematics Subject Classification. 11B68, 33C45.

Key words and phrases. Generalized Bernoulli polynomials, Generalized ApostolBernoulli polynomials, Generalized Apostol-Euler polynomials, Generating Functions, Addition Theorem, Special Functions. 
The literature contains a large number of interesting properties and relationships involving these polynomials $[1,5,6,8,21]$. These appear in many applications in combinatorics, number theory and numerical analysis.

Recently, some interesting analogues of the classical Bernoulli polynomials and the classical Euler polynomials have been investigated. Q.-M. Luo and H.M. Srivastava [18, 20] introduced the generalized ApostolBernoulli polynomials $\mathfrak{B}_{n}^{(\alpha)}(x ; \lambda)$ of order $\alpha \in \mathbb{C}$ (the case $\alpha=1$ was investigated first by T.M. Apostol [2, Eq. (3.1), p. 165]). In 2006, Q.-M. Luo [11] invented the generalized Apostol-Euler polynomials $\mathfrak{E}_{n}^{(\alpha)}(x ; \lambda)$ of order $\alpha \in \mathbb{C}$. Many authors have investigated these polynomials and numerous very interesting papers can be found in the literature. The reader can read (see, for examples, $[3,4,7,12,13,14,15,16,19,23]$ ).

Especially, the generalized Apostol-Bernoulli polynomials and the generalized Apostol-Euler polynomials are defined respectively as follows.

Definition 1.1. The generalized Apostol-Bernoulli polynomials $\mathfrak{B}_{n}^{(\alpha)}$ $(x ; \lambda)$ of order $\alpha \in \mathbb{C}$ are defined by means of the following generating function:

$$
\begin{gathered}
\left(\frac{t}{\lambda \mathrm{e}^{t}-1}\right)^{\alpha} \cdot \mathrm{e}^{x t}=\sum_{k=0}^{\infty} \mathfrak{B}_{k}^{(\alpha)}(x ; \lambda) \frac{t^{k}}{k !} \\
\left(|t|<2 \pi \text { when } \lambda=1 ;|t|<|\log \lambda| \text { when } \lambda \neq 1 ; 1^{\alpha}:=1\right)
\end{gathered}
$$

with

$$
B_{n}^{(\alpha)}(x)=\mathfrak{B}_{n}^{(\alpha)}(x ; 1) .
$$

Definition 1.2 (Luo [11]). The generalized Apostol-Euler polynomials $\mathfrak{E}_{n}^{(\alpha)}(x ; \lambda)$ of order $\alpha$ are defined by means of the following generating function:

$$
\left(\frac{2}{\lambda \mathrm{e}^{t}+1}\right)^{\alpha} \cdot \mathrm{e}^{x t}=\sum_{k=0}^{\infty} \mathfrak{E}_{k}^{(\alpha)}(x ; \lambda) \frac{t^{k}}{k !} \quad\left(|t|<|\log (-\lambda)| ; 1^{\alpha}:=1\right)
$$

with

$$
E_{n}^{(\alpha)}(x)=\mathfrak{E}_{n}^{(\alpha)}(x ; 1) .
$$

Kurt [9] gave a new generalization of the Bernoulli polynomials of order $\alpha$ in the following form. 
Definition 1.3. The generalized Bernoulli polynomials $B_{n}^{[m-1, \alpha]}(x)$ of order $\alpha \in \mathbb{C}, m \in \mathbb{N}$, are defined, in a suitable neighborhood of $t=0$, by means of the generating function

$$
\left(\frac{t^{m}}{\mathrm{e}^{t}-\sum_{l=0}^{m-1} \frac{t^{l}}{l !}}\right)^{\alpha} \cdot \mathrm{e}^{x t}=\sum_{k=0}^{\infty} B_{k}^{[m-1, \alpha]}(x) \frac{t^{k}}{k !} .
$$

Recently, the authors [29] investigated a new class of generalized Apostol-Bernoulli polynomials. These are defined as follows.

Definition 1.4. The generalized Apostol-Bernoulli polynomials $\mathfrak{B}_{n}^{[m-1, \alpha]}(x ; \lambda)$ of order $\alpha \in \mathbb{C}, m \in \mathbb{N}$, are defined, in a suitable neighborhood of $t=0$, with $|t+\log \lambda|<2 \pi$ by means of the generating function

$$
\left(\frac{t^{m}}{\lambda \mathrm{e}^{t}-\sum_{l=0}^{m-1} \frac{t^{l}}{l !}}\right)^{\alpha} \cdot \mathrm{e}^{x t}=\sum_{k=0}^{\infty} \mathfrak{B}_{k}^{[m-1, \alpha]}(x ; \lambda) \frac{t^{k}}{k !} .
$$

It is easy to see that if we set $m=1$ in (1.8), we arrive at

$$
\left(\frac{t}{\lambda \mathrm{e}^{t}-1}\right)^{\alpha} \cdot \mathrm{e}^{x t}=\sum_{k=0}^{\infty} \mathfrak{B}_{k}^{[0, \alpha]}(x ; \lambda) \frac{t^{k}}{k !} .
$$

This is the generating function for the generalized Apostol-Bernoulli polynomials of order $\alpha$. Thus, we have

$$
\mathfrak{B}_{n}^{[0, \alpha]}(x ; \lambda)=\mathfrak{B}_{n}^{(\alpha)}(x ; \lambda) .
$$

Obviously, when $\lambda=1$ in (1.8), we obtain (1.7). Moreover, if $m=1$ and $\alpha=1$, we obtain

$$
\mathfrak{B}_{n}^{[0,1]}(x ; 1)=B_{n}(x)
$$

where $B_{n}(x)$ are the classical Bernoulli polynomials.

Also, Srivastava et al. [26] introduced a new interesting class of ApostolBernoulli polynomials that are closely related to the new class that we present in this paper. They investigated the following form.

Definition 1.5. Let $a, b, c \in \mathbb{R}^{+}(a \neq b)$ and $n \in \mathbb{N}_{0}$. Then the generalized Bernoulli polynomials $\mathfrak{B}_{n}^{(\alpha)}(x ; \lambda ; a, b, c)$ of order $\alpha \in \mathbb{C}$ are defined by the following generating function

$$
\left(\frac{t}{\lambda b^{t}-a^{t}}\right)^{\alpha} \cdot c^{x t}=\sum_{n=0}^{\infty} \mathfrak{B}_{n}^{(\alpha)}(x ; \lambda ; a, b, c) \frac{t^{n}}{n !}
$$




$$
\left(\left|t \log \left(\frac{b}{a}\right)+\log \lambda\right|<2 \pi ; 1^{\alpha}:=1 ; x \in \mathbb{R}\right),
$$

where $\mathbb{N}_{0}:=\mathbb{N} \cup\{0\}, \mathbb{N}$ denotes the set of positive integers and $\mathbb{R}^{+}$the set of positive real numbers. It is easy to see that setting $a=1$ and $b=c=e$ in (1.12) would lead us to the Apostol-Bernoulli polynomials $\mathfrak{B}_{n}^{(\alpha)}(x ; \lambda)$ defined by (1.3). The case where $\alpha=1$ has been studied by Luo et al. [17].

The authors in [29] also gave a generalization of the Srivastava-Pintér addition theorem (see [28, Theorem 1, p. 379]) given in Theorem 1.6 below.

Theorem 1.6. The following relationship:

$$
\mathfrak{B}_{n}^{[m-1, \alpha]}(x+y ; \lambda)=
$$

$$
\sum_{k=0}^{n}\left(\begin{array}{c}
n \\
k
\end{array}\right)\left[\mathfrak{B}_{k}^{[m-1, \alpha]}(y ; \lambda)+\frac{k}{2} \sum_{j=0}^{k-1}\left(\begin{array}{c}
k-1 \\
j
\end{array}\right) \mathfrak{B}_{j}^{[m-1, \alpha]}(y ; \lambda) \mathfrak{B}_{k-1-j}^{(-1)}(0 ; \lambda)\right] \mathfrak{E}_{n-k}(x ; \lambda)
$$

$$
\left(\alpha, \lambda \in \mathbb{C} ; n \in \mathbb{N}_{0}\right)
$$

holds between the new class of generalized Apostol-Bernoulli polynomials and the Apostol-Euler polynomials.

In this paper, we propose a further generalization of the Bernoulli and the Apostol-Bernoulli polynomials and we give some properties involving them. For the new class of Bernoulli polynomials, we establish a new addition theorem with the help of a result given by Srivastava et al. [27]. We also give an extension of the Srivastava-Pintér theorem. Finally, we exhibit some relationships between the special case of the generalized Apostol-Bernoulli polynomials and other polynomials or special functions with the help of the new addition formula.

\section{An addition theorem for a new class of generalized Bernoulli polynomials}

In this section, we introduce a new class of generalized Bernoulli polynomials. We give some properties of that new class of polynomials and finally, we establish a new addition theorem based on a result due to Srivastava et al. [27].

Definition 2.1. For arbitrary complex parameter $\alpha$ and for $b, c$ $\in \mathbb{R}^{+}$, the generalized Bernoulli polynomials $B_{n}^{[m-1, \alpha]}(x, b, c), m \in \mathbb{N}$, 
are defined, in a suitable neighborhood of $t=0$, with $|t \log b|<2 \pi$ by means of the generating function

$$
\left(\frac{t^{m}}{b^{t}-\sum_{l=0}^{m-1} \frac{(t \log b)^{l}}{l !}}\right)^{\alpha} \cdot c^{x t}=\sum_{k=0}^{\infty} B_{k}^{[m-1, \alpha]}(x, b, c) \frac{t^{k}}{k !} .
$$

If we set $b=c=\mathrm{e}$ in (2.1), we find (1.7). Thus, we have

$$
B_{n}^{[m-1, \alpha]}(x, \mathrm{e}, \mathrm{e})=B_{n}^{[m-1, \alpha]}(x) .
$$

Moreover, if $b=c=\mathrm{e}, m=1$ and $\alpha=1$ in (2.1), we obtain

$$
B_{n}^{[0,1]}(x, \mathrm{e}, \mathrm{e})=B_{n}(x)
$$

where $B_{n}(x)$ are the classical Bernoulli polynomials.

Theorem 2.2. Let $b, c \in \mathbb{R}^{+}$and $m \in \mathbb{N}$. Then, the following relation

$$
x^{n}=\frac{(\ln b)^{m}}{(\ln c)^{n}} \sum_{k=0}^{n}\left(\begin{array}{l}
n \\
k
\end{array}\right) \frac{k !(\ln b)^{k}}{(k+m) !} B_{n}^{[m-1,1]}(x, b, c)
$$

holds true.

Proof. From (2.1) with $\alpha=1$, we have

$$
c^{x t}=\sum_{h=m}^{\infty} \frac{(\log b)^{h} t^{h-m}}{h !} \sum_{n=0}^{\infty} B_{n}^{[m-1,1]}(x, b, c) \frac{t^{n}}{n !} .
$$

Using the fact that

$$
c^{x t}=\mathrm{e}^{x t \log c}=\sum_{n=0}^{\infty}(x \log c)^{n} \frac{t^{n}}{n !},
$$

we have

$$
\sum_{n=0}^{\infty}(x \log c)^{n} \frac{t^{n}}{n !}=\sum_{h=m}^{\infty} \frac{(\log b)^{h} t^{h-m}}{h !} \sum_{n=0}^{\infty} B_{n}^{[m-1,1]}(x, b, c) \frac{t^{n}}{n !} .
$$

Some elementary manipulations give

$$
\sum_{n=0}^{\infty}(x \log c)^{n} \frac{t^{n}}{n !}=\sum_{n=0}^{\infty} \sum_{h=0}^{n}\left(\begin{array}{l}
n \\
h
\end{array}\right) \frac{(\log b)^{h+m} h !}{(h+m) !} B_{n-h}^{[m-1,1]}(x, b, c) \frac{t^{n}}{n !} .
$$

Comparing the coefficients of (2.8), the result follows easily. 
If we put $b=c=\mathrm{e}$ in (2.4), we find the result given by Natalini and Bernardini $[22$, p. 158, (2.6)], namely

$$
x^{n}=\sum_{h=0}^{n}\left(\begin{array}{l}
n \\
h
\end{array}\right) \frac{h !}{(h+m) !} B_{n-h}^{[m-1,1]}(x)
$$

where $B_{n-h}^{[m-1,1]}(x)$ are the Bernoulli polynomials defined by (1.7).

Moreover, if we set $b=c=\mathrm{e}$ and $m=1$ in (2.4), we obtain the following well-known expression (see [21, p. 26])

$$
x^{n}=\frac{1}{n+1} \sum_{h=0}^{n}\left(\begin{array}{c}
n+1 \\
k
\end{array}\right) B_{h}(x)
$$

where $B_{h}(x)$ are the classical Bernoulli polynomials defined by (1.1) with $\alpha=1$.

The generalized Bernoulli polynomials $B_{n}^{[m-1, \alpha]}(x, b, c)$ defined by (2.1) possess the following interesting properties.

Theorem 2.3. Let $b, c \in \mathbb{R}^{+}, \alpha$ an arbitrary complex number and $m \in \mathbb{N}$. Then, the generalized Bernoulli polynomials $B_{n}^{[m-1, \alpha]}(x, b, c)$ satisfy the following relations:

$$
B_{n}^{[m-1, \alpha+\beta]}(x+y, b, c)=\sum_{k=0}^{n}\left(\begin{array}{l}
n \\
k
\end{array}\right) B_{k}^{[m-1, \alpha]}(x, b, c) B_{n-k}^{[m-1, \beta]}(y, b, c)
$$

and

$$
B_{n}^{[m-1, \alpha]}(x+y, b, c)=\sum_{k=0}^{n}\left(\begin{array}{l}
n \\
k
\end{array}\right) B_{k}^{[m-1, \alpha]}(x, b, c)(y \log c)^{n-k} .
$$

Proof. Considering the generating function (2.1), the addition formulas (2.11) and (2.12) are easily deduced.

The next theorem has been invented by Srivastava et al. [27]. However, the theorem is given without proof (see, [27, p. 438-440]).

Theorem 2.4. Let $B(z)$ and $z^{-1} C(z)$ be arbitrary functions which are analytic in the neighborhood of the origin, and assume (for sake of simplicity) that

$$
B(0)=C^{\prime}(0)=1 .
$$


Define the sequence of functions $\left\{f_{n}^{(\alpha)}(x)\right\}_{n=0}^{\infty}$ by means of

$$
\sum_{n=0}^{\infty} f_{n}^{(\alpha)}(x) \frac{z^{n}}{n !}=[B(z)]^{\alpha} \exp (x C(z)),
$$

where $\alpha$ and $x$ are arbitrary complex numbers independent of $z$. Then, for arbitrary parameters $\lambda$ and $y$,

$$
f_{n}^{(\alpha+\lambda \gamma)}(x+\gamma y)=\sum_{k=0}^{n} \frac{\gamma+n}{\gamma+k}\left(\begin{array}{l}
n \\
k
\end{array}\right) f_{k}^{(\alpha-\lambda k)}(x-k y) f_{n-k}^{(\lambda k+\lambda \gamma)}(k y+\gamma y),
$$

provided that $\operatorname{Re}(\gamma)>0$.

Remark 2.5. The choice of 1 in the conditions of (2.13) is merely a convenient one. In fact, any nonzero constant values may be assumed for $B(0)$ and $C^{\prime}(0)$.

Now, applying the last theorem with special choices of functions and parameters furnishes the next very interesting addition formula. This formula is contained in the following corollary.

Corollary 2.6. Let $b, c \in \mathbb{R}^{+}$and $m \in \mathbb{N}$. Then, for arbitrary complex parameters $\alpha, \lambda, x$ and $y$, the generalized Bernoulli polynomials $B_{n}^{[m-1, \alpha]}(x, b, c)$ satisfy the addition formula

$$
B_{n}^{[m-1, \alpha+\lambda \gamma]}(x+\gamma y, b, c)=
$$

$$
\sum_{k=0}^{n} \frac{\gamma+n}{\gamma+k}\left(\begin{array}{l}
n \\
k
\end{array}\right) B_{k}^{[m-1, \alpha-\lambda k]}(x-k y, b, c) B_{n-k}^{[m-1, \lambda k+\lambda \gamma]}(k y+\gamma y, b, c)
$$

provided that $\operatorname{Re}(\gamma)>0$.

Proof. Setting $B(z)=\frac{t^{m}}{b^{t}-\sum_{l=0}^{m-1} \frac{(t \log b)^{l}}{l !}}$ and $C(z)=t \log c$ in Theorem 2.4, the result follows.

Moreover, if we set $\lambda=0$ in (2.16), we obtain

$$
B_{n}^{[m-1, \alpha]}(x+\gamma y, b, c)
$$


$(2.17)$

$$
\begin{aligned}
& =\sum_{k=0}^{n} \frac{\gamma+n}{\gamma+k}\left(\begin{array}{l}
n \\
k
\end{array}\right) B_{k}^{[m-1, \alpha]}(x-k y, b, c) B_{n-k}^{[m-1,0]}(k y+\gamma y, b, c) \\
& =\sum_{k=0}^{n} \frac{\gamma+n}{\gamma+k}\left(\begin{array}{l}
n \\
k
\end{array}\right) B_{k}^{[m-1, \alpha]}(x-k y, b, c)(\gamma+k)^{n-k}(y \log c)^{n-k} .
\end{aligned}
$$

The last result (2.17) will be very useful in the last section of this paper.

\section{A new class of generalized Apostol-Bernoulli polynomials}

The following definition provides a natural generalization and unification of the Apostol-Bernoulli polynomials $\mathfrak{B}_{n}^{[m-1, \alpha]}(x ; \lambda), m \in \mathbb{N}$, of order $\alpha \in \mathbb{C}$ introduced by R. Tremblay et al. [29] and those defined by (2.1).

Definition 3.1. For arbitrary real or complex parameter $\alpha$ and for $b$, $c \in \mathbb{R}^{+}$, the generalized Apostol-Bernoulli polynomials $\mathfrak{B}_{n}^{[m-1, \alpha]}(x, b, c ; \lambda)$, $m \in \mathbb{N}$, are defined, in a suitable neighborhood of $t=0$, with $\mid t \log b+$ $\log \lambda \mid<2 \pi$ by means of the generating function

$$
\left(\frac{t^{m}}{\lambda b^{t}-\sum_{l=0}^{m-1} \frac{(t \log b)^{l}}{l !}}\right)^{\alpha} \cdot c^{x t}=\sum_{k=0}^{\infty} \mathfrak{B}_{n}^{[m-1, \alpha]}(x, b, c ; \lambda) \frac{t^{k}}{k !} .
$$

It is easy to see that if we set $m=1, b=c=\mathrm{e}$ in (3.1), we arrive at

$$
\left(\frac{t}{\lambda \mathrm{e}^{t}-1}\right)^{\alpha} \cdot \mathrm{e}^{x t}=\sum_{k=0}^{\infty} \mathfrak{B}_{n}^{[0, \alpha]}(x, \mathrm{e}, \mathrm{e} ; \lambda) \frac{t^{k}}{k !} .
$$

This is the generating function for the generalized Apostol-Bernoulli polynomials of order $\alpha$. Thus, we have

$$
\mathfrak{B}_{n}^{[0, \alpha]}(x, \mathrm{e}, \mathrm{e} ; \lambda)=\mathfrak{B}_{n}^{(\alpha)}(x ; \lambda) .
$$

Obviously, when $\lambda=1$ in (3.1), we obtain (2.1). Moreover, if $m=1$ and $\alpha=1$, we obtain

$$
\mathfrak{B}_{n}^{[0,1]}(x, \mathrm{e}, \mathrm{e} ; 1)=B_{n}(x)
$$

where $B_{n}(x)$ are the classical Bernoulli polynomials.

The generalized Apostol-Bernoulli polynomials $\mathfrak{B}_{n}^{[m-1, \alpha]}(x, b, c ; \lambda)$ defined by (3.1) possess the following interesting properties that will be useful in the sequel. These are stated as Theorems 3.2 and 3.3 below. 
Theorem 3.2. Let $b, c \in \mathbb{R}^{+}, \alpha$ an arbitrary complex number and $m \in \mathbb{N}$. Then, the generalized Bernoulli polynomials $\mathfrak{B}_{n}^{[m-1, \alpha]}(x, b, c ; \lambda)$ satisfy the following relations:

$\mathfrak{B}_{n}^{[m-1, \alpha+\beta]}(x+y, b, c ; \lambda)=\sum_{k=0}^{n}\left(\begin{array}{l}n \\ k\end{array}\right) \mathfrak{B}_{k}^{[m-1, \alpha]}(x, b, c ; \lambda) \mathfrak{B}_{n-k}^{[m-1, \beta]}(y, b, c ; \lambda)$,

and

$$
\mathfrak{B}_{n}^{[m-1, \alpha]}(x+y, b, c ; \lambda)=\sum_{k=0}^{n}\left(\begin{array}{l}
n \\
k
\end{array}\right) \mathfrak{B}_{k}^{[m-1, \alpha]}(x, b, c ; \lambda)(y \log c)^{n-k} .
$$

Proof. Considering the generating function (3.1), the addition formulas (3.5) and (3.6) are easily deduced.

Theorem 3.3. The generalized Apostol-Bernoulli polynomials $\mathfrak{B}_{n}^{[m-1, \alpha]}$ $(x, b, c ; \lambda)$ satisfy the following recurrence relation:

$$
\begin{gathered}
\lambda \mathfrak{B}_{n}^{[m-1, \alpha]}(x+1, b, c ; \lambda)-\mathfrak{B}_{n}^{[m-1, \alpha]}(x, b, c ; \lambda)= \\
n \sum_{k=0}^{n-1}\left(\begin{array}{c}
n-1 \\
k
\end{array}\right) \mathfrak{B}_{k}^{[m-1, \alpha]}(x, b, c ; \lambda) \mathfrak{B}_{n-1-k}^{(-1)}(0 ; \lambda ; 1, c, a)
\end{gathered}
$$

where $\mathfrak{B}_{n-1-k}^{(-1)}(0 ; \lambda ; 1, c, a)$ are the generalized Apostol-Bernoulli polynomials defined by (1.12).

Proof. Considering the expression $\lambda \mathfrak{B}_{n}^{[m-1, \alpha]}(x+1, b, c ; \lambda)-\mathfrak{B}_{n}^{[m-1, \alpha]}$ $(x, b, c ; \lambda)$ and using the generating functions (2.1) and (1.12), (3.7) follows easily.

Remark 3.4. Setting $m=1$ and $b=c=$ e in (3.7) and with the help of (3.3), we find

$\lambda \mathfrak{B}_{n}^{(\alpha)}(x+1 ; \lambda)-\mathfrak{B}_{n}^{(\alpha)}(x ; \lambda)=n \sum_{k=0}^{n-1}\left(\begin{array}{c}n-1 \\ k\end{array}\right) \mathfrak{B}_{k}^{(\alpha)}(x ; \lambda) \mathfrak{B}_{n-1-k}^{(-1)}(0 ; \lambda)$.

Using the well known result (see, [20])

$$
\mathfrak{B}_{n}^{(\alpha+\beta)}(x+y ; \lambda)=\sum_{k=0}^{n}\left(\begin{array}{l}
n \\
k
\end{array}\right) \mathfrak{B}_{k}^{(\alpha)}(x ; \lambda) \mathfrak{B}_{n-k}^{(\beta)}(y ; \lambda),
$$

(3.8) becomes the familiar relation for the generalized Apostol-Bernoulli polynomials (see, [20])

$$
\lambda \mathfrak{B}_{n}^{(\alpha)}(x+1 ; \lambda)-\mathfrak{B}_{n}^{(\alpha)}(x ; \lambda)=n \mathfrak{B}_{n-1}^{(\alpha-1)}(x ; \lambda) .
$$




\section{A generalization and some analogues of the Srivastava- Pintér addition theorem}

In this section, we give a generalization of the Srivastava-Pintér addition theorem and an analogue. We end this section by giving two interesting relationships involving the new addition formula (2.17).

Theorem 4.1. The following relationship

$$
\mathfrak{B}_{n}^{[m-1, \alpha]}(x+y, b, c ; \lambda)=\sum_{k=0}^{n}\left(\begin{array}{l}
n \\
k
\end{array}\right) \mathfrak{E}_{n-k}(x ; \lambda)(\log c)^{n-k}
$$

$$
\begin{gathered}
\times\left[\mathfrak{B}_{k}^{[m-1, \alpha]}(y, b, c ; \lambda)+\frac{k}{2} \sum_{j=0}^{k-1}\left(\begin{array}{c}
k-1 \\
j
\end{array}\right) \mathfrak{B}_{j}^{[m-1, \alpha]}(y, b, c ; \lambda) \mathfrak{B}_{k-1-j}^{(-1)}(0 ; \lambda ; 1, c, a)\right] \\
\left(\alpha, \lambda \in \mathbb{C} ; n \in \mathbb{N}_{0}\right)
\end{gathered}
$$

holds between the new class of generalized Apostol-Bernoulli polynomials and the Apostol-Euler polynomials.

Proof. First of all, if we substitute the entry (9) for $x^{n}$ from Table 1 into the right-hand side of (3.6), we get

$$
\mathfrak{B}_{n}^{[m-1, \alpha]}(x+y, b, c ; \lambda)
$$

$$
\begin{aligned}
= & \frac{1}{2} \sum_{k=0}^{n}\left(\begin{array}{l}
n \\
k
\end{array}\right) \mathfrak{B}_{k}^{[m-1, \alpha]}(y, b, c ; \lambda)(\log c)^{n-k}\left[\mathfrak{E}_{n-k}(x ; \lambda)+\lambda \sum_{j=0}^{n-k}\left(\begin{array}{c}
n-k \\
j
\end{array}\right) \mathfrak{E}_{j}(x ; \lambda)\right] \\
= & \frac{1}{2} \sum_{k=0}^{n}\left(\begin{array}{l}
n \\
k
\end{array}\right) \mathfrak{B}_{k}^{[m-1, \alpha]}(y, b, c ; \lambda)(\log c)^{n-k} \mathfrak{E}_{n-k}(x ; \lambda) \\
& +\frac{\lambda}{2} \sum_{k=0}^{n}\left(\begin{array}{l}
n \\
k
\end{array}\right) \mathfrak{B}_{k}^{[m-1, \alpha]}(y, b, c ; \lambda)(\log c)^{n-k} \sum_{j=0}^{n-k}\left(\begin{array}{c}
n-k \\
j
\end{array}\right) \mathfrak{E}_{j}(x ; \lambda),
\end{aligned}
$$

which, upon inverting the order of summation and using the following elementary combinatorial identity:

$$
\left(\begin{array}{c}
m \\
l
\end{array}\right)\left(\begin{array}{c}
l \\
n
\end{array}\right)=\left(\begin{array}{c}
m \\
n
\end{array}\right)\left(\begin{array}{c}
m-n \\
m-l
\end{array}\right) \quad\left(m \geq l \geq n ; l, m, n \in \mathbb{N}_{0}\right),
$$

yields

$$
\mathfrak{B}_{n}^{[m-1, \alpha]}(x+y, b, c ; \lambda)=\frac{1}{2} \sum_{k=0}^{n}\left(\begin{array}{l}
n \\
k
\end{array}\right) \mathfrak{B}_{k}^{[m-1, \alpha]}(y, b, c ; \lambda) \mathfrak{E}_{n-k}(x ; \lambda)(\log c)^{n-k}
$$


(4.4)

$$
+\frac{\lambda}{2} \sum_{j=0}^{n}\left(\begin{array}{c}
n \\
j
\end{array}\right) \mathfrak{E}_{j}(x ; \lambda)(\log c)^{j} \sum_{k=0}^{n-j}\left(\begin{array}{c}
n-j \\
k
\end{array}\right) \mathfrak{B}_{k}^{[m-1, \alpha]}(y, b, c ; \lambda)(\log c)^{n-j-k} .
$$

The innermost sum in (4.4) can be calculated with the help of (3.6) with, of course,

$$
x=1 \quad \text { and } \quad n \mapsto n-j \quad\left(0 \leq j \leq n ; n, j \in \mathbb{N}_{0}\right) .
$$

We thus find from (4.4) that

(4.5)

$$
\begin{aligned}
\mathfrak{B}_{n}^{[m-1, \alpha]}(x+y, b, c ; \lambda) & =\frac{1}{2} \sum_{k=0}^{n}\left(\begin{array}{l}
n \\
k
\end{array}\right) \mathfrak{B}_{k}^{[m-1, \alpha]}(y, b, c ; \lambda) \mathfrak{E}_{n-k}(x ; \lambda)(\log c)^{n-k} \\
& +\frac{\lambda}{2} \sum_{j=0}^{n}\left(\begin{array}{c}
n \\
n-j
\end{array}\right) \mathfrak{B}_{n-j}^{[m-1, \alpha]}(y+1, b, c ; \lambda) \mathfrak{E}_{j}(x ; \lambda)(\log c)^{j} \\
& =\frac{1}{2} \sum_{k=0}^{n}\left(\begin{array}{l}
n \\
k
\end{array}\right)\left[\mathfrak{B}_{k}^{[m-1, \alpha]}(y, b, c ; \lambda)+\lambda \mathfrak{B}_{k}^{[m-1, \alpha]}(y+1, b, c ; \lambda)\right] \\
& \times \mathfrak{E}_{n-k}(x ; \lambda)(\log c)^{n-k}
\end{aligned}
$$

which, with the relation (3.7), leads us to the relationship (4.1) asserted by Theorem 4.1 .

Remark 4.2. If we put $\lambda=1$ and $b=c=\mathrm{e}$ in Theorem 4.1, we obtain Theorem 1.6. Furthermore, if we set $m=1$, we recover the Srivastava-Pintér addition theorem [28]. 


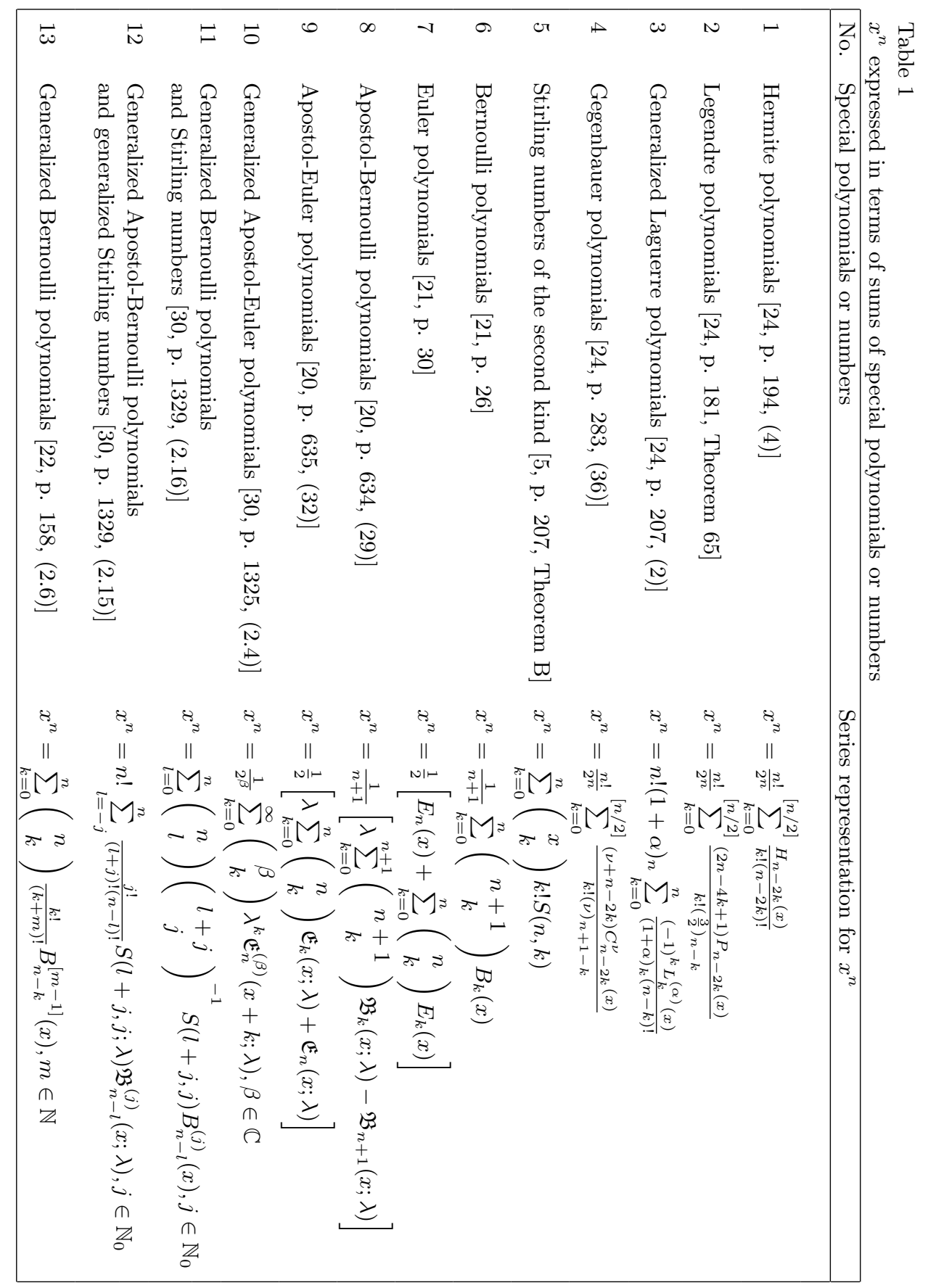


With the help of Table 1 that contains a list of series representation for $x^{n}$ in terms of special polynomials or numbers, we can find some analogues of the Srivastava-Pintér addition theorem. As example, if we consider the entry 3 , we obtain the next theorem.

Theorem 4.3. The following relationship (4.6)

$$
\begin{aligned}
\mathfrak{B}_{n}^{[m-1, \alpha]}(x+y, b, c ; \lambda)= & \sum_{k=0}^{n}\left(\begin{array}{l}
n \\
k
\end{array}\right) \mathfrak{B}_{k}^{[m-1, \alpha]}(y, b, c ; \lambda)(\log c)^{n-k}(n-k) !(1+\alpha)_{n-k} \\
& \times \sum_{j=0}^{n-k} \frac{(-1)^{j} L_{j}^{(\alpha)}(x)}{(1+\alpha)_{j}(n-k-j) !}
\end{aligned}
$$

holds between the new class of generalized Apostol-Bernoulli polynomials and the generalized Laguerre polynomials defined by

$$
(1-t)^{-\alpha-1} \mathrm{e}^{\left(\frac{x t}{t-1}\right)}=\sum_{n=0}^{\infty} L_{n}^{(\alpha)}(x) t^{n} .
$$

Proof. From the addition theorem (3.6) and entry 3.

Now, let's shift our focus on two special cases of the addition theorem (2.17) involving the new class of generalized Bernoulli polynomials defined by (2.1). These are contained in the two next theorems.

Theorem 4.4. The following relationship (4.8)

$$
\begin{aligned}
B_{n}^{[m-1, \alpha]}(x+\gamma y, b, c)= & \sum_{k=0}^{n} \frac{\gamma+n}{\gamma+k}\left(\begin{array}{l}
n \\
k
\end{array}\right) B_{k}^{[m-1, \alpha]}(x-k y, b, c)(\gamma+k)^{n-k}(\log c)^{n-k} \\
& \times \sum_{j=0}^{n-k}\left(\begin{array}{c}
y \\
j
\end{array}\right) j ! S(n-k, j)
\end{aligned}
$$

holds between the new class of generalized Bernoulli polynomials and the Stirling numbers of the second kind that could be computed by the formula [25, p. 58, Eq. 1.5]

$$
S(n, k)=\frac{1}{k !} \sum_{j=0}^{k}(-1)^{k-j}\left(\begin{array}{l}
k \\
j
\end{array}\right) j^{n} .
$$

Proof. From the addition theorem (2.17) and entry 5. 
Theorem 4.5. The following relationship (4.10)

$$
\begin{aligned}
B_{n}^{[m-1, \alpha]}(x+\gamma y, b, c)= & \sum_{k=0}^{n} \frac{\gamma+n}{\gamma+k}\left(\begin{array}{l}
n \\
k
\end{array}\right) B_{k}^{[m-1, \alpha]}(x-k y, b, c)(\gamma+k)^{n-k}(\log c)^{n-k} \\
& \times \sum_{j=0}^{n-k}\left(\begin{array}{c}
n-k \\
j
\end{array}\right) \frac{j !}{(j+m) !} B_{n-k-j}^{[m-1]}(y)
\end{aligned}
$$

holds between the new class of generalized Bernoulli polynomials and the generalized Bernoulli polynomials defined by (1.7).

Proof. From the addition theorem (2.17) and entry 13.

We conclude this paper by noting that we can apply theorem 2.4 to the class of generalized Bernoulli polynomials defined by (1.12) when $\lambda=1$. This gives the following new addition formula for that class of polynomials.

Theorem 4.6. The following addition formula

$$
\mathfrak{B}_{n}^{(\alpha)}(x+\gamma y ; 1 ; a, b, c)
$$

$$
\begin{aligned}
& =\sum_{k=0}^{n} \frac{\gamma+n}{\gamma+k}\left(\begin{array}{l}
n \\
k
\end{array}\right) \mathfrak{B}_{k}^{(\alpha)}(x-k y ; 1 ; a, b, c) \mathfrak{B}_{n-k}^{(0)}(k y+\gamma y ; 1 ; a, b, c) \\
& =\sum_{k=0}^{n} \frac{\gamma+n}{\gamma+k}\left(\begin{array}{l}
n \\
k
\end{array}\right) \mathfrak{B}_{k}^{(\alpha)}(x-k y ; 1 ; a, b, c)(\gamma+k)^{n-k}(y \log c)^{n-k} .
\end{aligned}
$$

holds true for the generalized Bernoulli polynomials defined by (1.12).

Proof. Setting $B(z)=\frac{t}{b^{t}-a^{t}}$ and $C(z)=t \log c$ in Theorem 2.4, the result follows.

It could be interesting to consider this addition formula (4.11) in conjunction with series representation involving some special functions for $x^{n}$ in order to derive some analogues of the Srivastava-Pintér addition theorem. A paper dealing with analogues of the Srivastava-Pintér addition theorem and addition theorem (Theorem 2.4) for two new classes of polynomials, that is, the generalized Apostol-Euler polynomials and the generalized Apostol-Genocchi polynomials is under preparation. 


\section{References}

1. M. Abramowitz and I. A. Stegun, Handbook of mathematical functions with formulas, graphs and mathematical tables, National Bureau of Standards, Washington, DC, 1964.

2. T. M. Apostol, On the Lerch zeta function, Pacific J. Math. 1 (1951), 161-167.

3. K. N. Boyadzhiev, Apostol-bernoulli functions, derivative polynomials and eulerian polynomials, Advances and Applications in Discrete Mathematics 1 (2008), no. $2,109-122$.

4. J. Choi, P. J. Anderson, and H. M. Srivastava, Some q-extensions of the apostolbernoulli and the apostol-euler polynomials of order $n$, and the multiple hurwitz zeta function, Appl. Math. Comput 199 (2008), 723-737.

5. L. Comtet, Advanced combinatorics: The art of finite and infinite expansions, (Translated from french by J.W. Nienhuys), Reidel, Dordrecht, 1974.

6. A. Erdelyi, W. Magnus, F. Oberhettinger, and F. Tricomi, Higher transcendental functions, vols.1-3, 1953.

7. M. Garg, K. Jain, and H. M. Srivastava, Some relationships between the generalized apostol-bernoulli polynomials and hurwitz-lerch zeta functions, Integral Transform Spec. Funct. 17 (2006), no. 11, 803-815.

8. E. R. Hansen, A table of series and products, Prentice-Hall, Englewood Cliffs, NJ, 1975

9. B. Kurt, A further generalization of the Bernoulli polynomials and on the 2DBernoulli polynomials $B_{n}^{2}(x, y)$, Appl. Math.Sci. Vol.4 (47) (2010), 2315-2322.

10. Y. Luke, The special functions and their approximations, vols. 1-2, 1969.

11. Q.-M. Luo, Apostol-Euler polynomials of higher order and gaussian hypergeometric functions, Taiwanese J. Math. 10 (4) (2006), 917-925.

12. - Fourier expansions and integral representations for the apostol-bernoulli and apostol-euler polynomials, Math. Comp. 78 (2009), 2193-2208.

13. _ The multiplication formulas for the apostol-bernoulli and apostol-euler polynomials of higher order, Integral Transform Spec. Funct. 20 (2009), 377-391.

14. Some formulas for apostol-euler polynomials associated with hurwitz zeta function at rational arguments, Applicable Analysis and Discrete Mathematics 3 (2009), 336-346.

15. _ An explicit relationship between the generalized apostol-bernoulli and apostol-euler polynomials associated with $\lambda$-stirling numbers of the second kind, Houston J. Math. 36 (2010), 1159-1171.

16. Extension for the genocchi polynomials and its fourier expansions and integral representations, Osaka J. Math. 48 (2011), 291-310.

17. Q.-M. Luo, B.-N. Guo, F. Qui, and L. Debnath, Generalizations of Bernoulli numbers and polynomials, Int. J. Math. Math. Sci. 59 (2003), 3769-3776.

18. Q.-M. Luo and H.M. Srivastava, Some generalizations of the Apostol-Bernoulli and Apostol-Euler polynomials, J. Math.Anal.Appl. 308 (1) (2005), 290-302.

19. Q.-M. Luo and H.M. Srivastava, Some generalizations of the apostol-genocchi polynomials and the stirling numbers of the second kind, Appl. Math. Comput 217 (2011), 5702-5728.

20. Q.M. Luo and H.M. Srivastava, Some relationships between the Apostol-Bernoulli and Apostol-Euler polynomials, Comput. Math. Appl. 51 (2006), 631-642. 
21. F. Magnus, W. Oberhettinger and R. P. Soni, Formulas and theorems for the special functions of mathematical physics, Third enlarged edition, Springer-Verlag, New York, 1966.

22. P. Natalini and A. Bernardini, A generalization of the Bernoulli polynomials, J. Appl. Math. 3 (2003), 155-163.

23. M. Prévost, Padé approximation and apostol-bernoulli and apostol-euler polynomials, J. Comput. Appl. Math. 233 (2010), 3005-3017.

24. E. D. Rainville, Special functions, Macmillan Company, New York, 1960.

25. H. M. Srivastava and J. Choi, Series associated with zeta and related functions, Kluwer Academin Publishers, Dordrecht, Boston and London, 2001.

26. H. M. Srivastava, M. Garg, and S. Choudhary, A new generalization of the Bernoulli and related polynomials, Russian J. Math. Phys. 17 (2010), 251-261.

27. H. M. Srivastava, J.-L. Lavoie, and R. Tremblay, A class of addition theorems, Canad. Math.Bull. 26 (1983), 438-445.

28. H. M. Srivastava and À. Pintér, Remarks on some relationships between the Bernoulli and Euler polynomials, Appl. Math. Lett. 17 (4) (2004), 375-380.

29. R. Tremblay, S. Gaboury, and B. J. Fugère, A new class of generalized ApostolBernoulli polynomials and some analogues of the Srivastava-Pintér addition theorem, Appl. Math. Lett. 24 (2011), 1888-1893.

30. W. Wang, C. Jia, and T. Wang, Some results on the Apostol-Bernoulli and Apostol-Euler polynomials, Comput. Math. Appl. 55 (2008), 1322-1332.

R. Tremblay

Department of Mathematics and Computer Science, University of Quebec at Chicoutimi,

Quebec G7H 2B1, Canada.

E-mail: rtrembla@uqac.ca

\section{S. Gaboury}

Department of Mathematics and Computer Science, University of Quebec at Chicoutimi,

Quebec G7H 2B1, Canada.

E-mail: s1gabour@uqac.ca

J. Fugère

Department of Mathematics and Computer Science, Royal Military College,

Ontario K7K 5L0, Canada.

E-mail: fugerej@rmc.ca 